\title{
Editorial
}

\section{Progress in laser acceleration of particles}

Laser acceleration of particles is currently a very active area of research in Plasma Physics, with an emphasis on acceleration of electrons and ions using short but intense laser pulses. In this special issue we access the current status of this field by inviting leading researchers all over the world to contribute their original works here. Many of these results were first presented at the recent Laser-Particle Acceleration Workshop (LPAW 2011) held in Wuzhen, China in June 2011. In addition to the laser wakefield acceleration (LWFA) of electrons (Tzoufras et al.) and laser acceleration of ions (Tsung et al.), there were exciting new proposals for a protondriven plasma wakefield accelerator (Xia et al.) and for a dielectric-structure-based two-beam accelerator (Gai et al.) presented at this workshop, and we are very pleased to have the authors' contributions on these included here.

In the laser wakefield area, exploration of the selfguided regime continues to be of interest with results reported from the University of Texas at Austin group on self-injected electron acceleration in a very lowdensity plasma. These are the first results from the Texas Petawatt laser on LWFA (Wang et al.) and the first to report self-injection at a density of $10^{17} \mathrm{~cm}^{-3}$. How electrons are injected into plasma wakefields continues to be a debated topic. Lotov's paper develops a theoretical model for side injection of electrons and calculates an optimum angle at which such injection occurs. The selfguided LWFA usually operates in the blowout or bubble regime where the focusing force on the accelerating electrons is expected to be enormous. In a paper by Tzoufras et al., the weakly nonlinear regime is exploited to propagate a laser pulse in plasma for hundreds of Rayleigh lengths to generate a $100-\mathrm{GeV}$ electron beam in a single stage of LWFA at an average gradient of $3.6 \mathrm{GeV} / \mathrm{m}$. Some researchers in the community believe that a LWFA with weak focusing force may be better for generating a lower emittance electron beam, and that one way to do this is to employ a super-Gaussian laser pulse (Ostermayr et al.). A completely different approach to generate multi-GeV energy range electrons is proposed by Wang et al. Their two-dimensional particle in cell (PIC) code simulations show that by using a laser pulse with an intensity of $10^{24}-10^{25} \mathrm{~W} \mathrm{~cm}^{-2}$ incident on a thin foil target, it is possible to generate 1-10$\mathrm{GeV}$ electrons. However, at even higher laser intensities, radiation damping becomes a major energy loss mechanism. High-resolution measurements of electron beam characteristics have been made by the Alpha-
X Collaboration (Welsh et al.). Using the well-known pepper-pot technique, this group has measured the transverse emittance of a $100+\mathrm{MeV}$ electron beam from a laser accelerator to be better than $2.2 \mathrm{~mm}$.mrad, which compares very well with conventional radio-frequency accelerators.

The laser acceleration of ions has been also rather extensively addressed in this special issue. When the laserwakefield-accelerated high-energy electrons escape the plasma, they create a space charge field at the exit of the wake. This sheath potential confines the lower energy electrons that return to the plasma in a vortex-like motion. This in turn produces a time-varying azimuthal $\mathrm{B}$ field and thus a time-varying $\mathrm{E}$ field. The sheath field and this time-varying $\mathrm{E}$ field are subsequently seen to accelerate ions in an experiment carried out by Lemos et al. Most of the ion acceleration experiments today are being done with either a Ti-sapphire laser, or with a 1-micron wavelength laser. The $\mathrm{CO}_{2}$ laser has some distinct advantages when it is used in conjunction with a gas jet target for ion acceleration (Tsung et al.). The critical density for the $\mathrm{CO}_{2}$ laser wavelength is $10^{19} \mathrm{~cm}^{-3}$. Since such densities are easily obtained in a gas jet target (that produces a debris-free plasma at a high rep rate), it is possible to generate a forward propagating, collimated beam of ions to inject into a conventional accelerator. This possibility is explored in a paper by Antici et al. They find that the final emittance of a laser-injected ion beam emerging from a conventional accelerator is mainly determined by the capturing optic and the accelerating structure.

In the next few years we will see LWFA-driven 50 $100-\mathrm{MeV}$ electron guns and laser-driven proton injectors for a conventional accelerator. Laser-driven electrons will find uses in coherent and incoherent soft-x-ray and $\mathrm{x}$-ray sources. With several petawatt class laser systems that are coming on line, LWFA-generated electrons are likely to approach $10-\mathrm{GeV}$ energy. The future for this area of research is very bright and we at the Journal of Plasma Physics are very pleased to bring you these latest developments in this rapidly evolving field.

Chan Joshi, UCLA, USA

Wei Lu, Tsinghua University, China

Zhengming Sheng, Shanghai Jiao Tong University,

China

Co-editors 


\section{References}

Antici, P., Migliorati, M., Mostacci, A., Picardi, L., Palumbo, L. and Ronsivalle, C. 2012 Sensitivity study in a compact accelerator for laser-generated protons. J. Plasma Phys. 78(4), 441-445.

Gai, W., Power, J. G. and Jing, C. 2012 Short-pulse dielectric two-beam acceleration. J. Plasma Phys. 78(4), 339345.

Lemos, N., Martins, J. L., Dias, J. M., Marsh, K. A., Pak, A. and Joshi, C. 2012 Forward directed ion acceleration in a LWFA with ionization-induced injection. J. Plasma Phys. 78(4), 327-331.

Lotov, K. V. 2012 Optimum angle for side injection of electrons into linear plasma wakefields. J. Plasma Phys. 78(4), 455459 .

Ostermayr, T. et al. 2012 Laser plasma accelerator driven by a super-Gaussian pulse. J. Plasma Phys. 78(4), 447-453.

Tsung, F., Tochitsky, S. Ya., Haberberger, D. J., Mori, W. B. and Joshi, C. $2012 \mathrm{CO}_{2}$ Laser acceleration of forward directed $\mathrm{MeV}$ proton beams in a gas target at critical plasma density. J. Plasma Phys. 78(4), 373382.
Tzoufras, M., Huang, C., Cooley, J. H., Tsung, F. S., Vieira, J. and Mori, W. B. 2012 Simulations of efficient laser wakefield accelerators from 1 to $100 \mathrm{GeV}$. J. Plasma Phys. 78(4), 401-412.

Welsh, G. H., Wiggins, S. M., Issac, R. C., Brunetti, E., Manahan, G. G., Islam, M. R., Cipiccia, S., Aniculaesei, C., Ersfeld, B. and Jaroszynski, D. A. 2012 High resolution electron beam measurements on the ALPHA-X laserplasma wakefield accelerator. J. Plasma Phys. 78(4), 393399.

Wang, X. et al. 2012 Self-injected petawatt laser-driven plasma electron acceleration in $10^{17} \mathrm{~cm}^{-3}$ plasma. J. Plasma Phys. 78(4), 413-419.

Wang, W.-M., Sheng, Z.-M., Kawata, S., Zheng, C.-Y., Li, Y.-T., Chen, L.-M., Dong, Q.-L., Lu, X., Ma, J.-L. and Zhang, J. 2012 Towards Sub-TeV electron beams driven by ultrashort, ultra-intense laser pulses. J. Plasma Phys. 78(4), 461-468.

Xia, G., Assmann, R., Fonseca, R. A., Huang, C., Mori, W., Silva, L. O., Vieira, J., Zimmermann, F. and Muggli, P. 2012 A proposed demonstration of an experiment of proton-driven plasma wakefield acceleration based on CERN SPS. J. Plasma Phys. 78(4), 347-353. 